\title{
CHARACTERISTIC, MAXIMUM MODULUS AND VALUE DISTRIBUTION
}

\author{
BY
}

W. K. HAYMAN AND J. F. ROSSI ${ }^{1}$

\begin{abstract}
Let $f$ be an entire function such that $\log M(r, f) \sim T(r, f)$ on a set $E$ of positive upper density. Then $f$ has no finite deficient values. In fact, if we assume that $E$ has density one and $f$ has nonzero order, then the roots of all equations $f(z)=a$ are equidistributed in angles. In view of a recent result of Murai [6] the conclusions hold in particular for entire functions with Fejér gaps.
\end{abstract}

1. Introduction. In a recent paper Murai [6] proved among other things that if $f(z)=\sum_{0}^{\infty} a_{n} z^{\lambda_{n}}$ is an entire function with Fejér gaps, i.e.

$$
\sum \lambda_{n}^{-1}<\infty,
$$

then $f(z)$ can have no deficient values. In the course of his proof Murai showed that for such a function

$$
T(r, f) \sim \log M(r, f)
$$

as $r \rightarrow \infty$ outside a set of finite logarithmic measure, where $T(r, f)$ is the Nevanlinna characteristic and $M(r, f)$ the maximum modulus of $f$. In this paper we show that the condition (1.2) suffices in order that a transcendental entire function should have no deficient values and, subject to certain growth conditions, that the roots of all equations $f(z)=a$ are equidistributed in angles. It is clear that some additional growth condition is necessary for this. In fact if $f(z)$ is an entire function of genus zero, $n(r)$ is the counting function of its zeros and

$$
N(r)=\int_{0}^{r} \frac{n(t) d t}{t}
$$

then $[4,(4.11)$, p. 133]

$$
n(r)=o\{N(r)\}
$$

implies (1.2), but (1.3) is unaffected by the arguments of the zeros. We shall see that a weaker gap condition than (1.1), namely Fabry gaps

$$
\lambda_{n} / n \rightarrow \infty,
$$

is sufficient or alternatively a growth condition, namely that $f(z)$ has positive order and satisfies (1.2) on a set of density one.

Received by the editors June 15, 1983 and, in revised form, August 29, 1983.

1980 Mathematics Subject Classification. Primary 30D35; Secondary 30D20.

${ }^{1}$ Research performed as a NATO Postdoctoral Fellow at Imperial College, London. 
2. Statement of results. We take for granted the usual notation of Nevanlinna theory. Let $f(z)$ be a transcendental entire function of order $\lambda$ and lower order $\mu$, where $0 \leqslant \mu \leqslant \lambda \leqslant \infty$.

THEOREM 1. Suppose that $f(z)$ is an entire function such that

$$
\lim \frac{T(r, f)}{\log M(r, f)}=1
$$

as $r \rightarrow \infty$ on a set $E$ of positive upper density $\delta$. Then there exists a set $F$ of density zero, such that for every complex a we have

$$
N(r, a, f) \sim T(r, f)
$$

as $r \rightarrow \infty$ in $E \backslash F$. In particular, $\delta(a, f)=0$ for every $a$.

We write $n\left(r, \theta_{1}, \theta_{2}, a\right)$ for the number of roots of the equation $f(z)=a$ in the sector

$$
S\left(r, \theta_{1}, \theta_{2}\right): 0<|z|<r, \quad \theta_{1}<\arg z<\theta_{2},
$$

and

$$
N\left(r, \theta_{1}, \theta_{2}, a\right)=\int_{0}^{r} \frac{n\left(t, \theta_{1}, \theta_{2}, a\right) d t}{t} .
$$

Our next result is

THEOREM 2. If $\lambda>0$ and $f(z)$ satisfies (2.1) as $r \rightarrow \infty$ on a set $E_{1}$ of density one, then there exists a set $E_{2}$ of upper density one such that

$$
N\left(r, \theta_{1}, \theta_{2}, a\right) \sim \frac{\theta_{2}-\theta_{1}}{2 \pi} T(r, f)
$$

as $r \rightarrow \infty$ on $E_{2}$ for every complex a and every pair $\theta_{1}, \theta_{2}$ such that $\theta_{1}<\theta_{2} \leqslant \theta_{1}+2 \pi$.

Our results have a natural extension to subharmonic functions when we consider the Riesz mass on set $G$ of a subharmonic function $u(z)$ to be the analogue of the number of zeros on $G$ of the function $f(z)-a$. We can then apply the subharmonic result to $u(z)=\log |f(z)-a|$, provided that the set $G$ is chosen independent of $a$.

3. A growth result for real functions. In order to obtain Theorems 1 and 2 we prove an extension of a growth lemma of Edrei and Fuchs [1] to entire functions of arbitrary growth. Such an extension is possible if we work with the maximum modulus instead of the characteristic. However, in order to do this we need a sharpened version of an inequality for real functions of Hayman and Stewart [5]. We assume in this section that $f(x)$ is a real function such that for sufficiently large positive $x, f^{(n-1)}(x)$ is convex. Thus for large $x, f^{(n)}(x)$ exists and is increasing outside a countable set. If, in addition, $f^{(n)}(x)>0$ for large $x$, we say that $f(x) \in B(n)$ and define

$$
f_{n}(x)=\inf _{h>0} \frac{f(x+h)}{h^{n}} .
$$


It was proved in [5] that for $f(x) \in B(n)$ we have, given $K>1$,

$$
f_{n}(x)<(e K / n)^{n} f^{(n)}(x)
$$

on a set $E$ of positive lower density. In this paper we need to prove that the lower density is close to one if $K$ is large. More precisely we have

THEOREM 3. If $E$ is the set of all $x$ for which (3.1) is true when $f(x) \in B(n)$, then if $\delta(E)$ denotes the lower density of $E$, we have $\delta(E) \geqslant(K-1) /(K-1+n)$.

We follow the argument of [5] and define

$$
\beta(x)=\sup _{0 \leqslant \nu \leqslant n-1}\left\{\frac{f^{(\nu)}(x)}{f^{(n)}(x)}\right\}^{1 /(n-\nu)} .
$$

We need

Lemma 1. If $f(x) \in B(n)$ and $f, f^{\prime}, \ldots, f^{(n)}$ are all positive for $x \geqslant x_{0}$, then $x-\beta(x)$ is increasing for $x \geqslant x_{0}$.

In [5, Lemma 3] it was shown that

$$
\beta\{x+\delta \beta(x)\} \leqslant e^{\delta} \beta(x) .
$$

Suppose that there exist $x_{1}$ and $x_{2}$, such that $x_{0} \leqslant x_{1}<x_{2}$ and $x_{2}-\beta\left(x_{2}\right)<x_{1}-$ $\beta\left(x_{1}\right)$. Then there exists $C>1$ such that $C x_{2}-\beta\left(x_{2}\right)=C x_{1}-\beta\left(x_{1}\right)$. We write for a large positive integer $N$

$$
h=\left(x_{2}-x_{1}\right) / N, \quad \xi_{j}=x_{1}+j h, \quad j=0, \ldots, N,
$$

and deduce that for at least one $j, 0 \leqslant j \leqslant N-1$, we have

$$
C \xi_{j+1}-\beta\left(\xi_{j+1}\right) \leqslant C \xi_{j}-\beta\left(\xi_{j}\right)
$$

i.e.

$$
\beta\left(\xi_{j}+h\right) \geqslant \beta\left(\xi_{j}\right)+C h=\beta\left(\xi_{j}\right)\left\{1+C h / \beta\left(\xi_{j}\right)\right\} .
$$

Writing $h=\delta \beta\left(\xi_{j}\right)$ we obtain

$$
\beta\left(\xi_{j}+h\right) \geqslant \beta\left(\xi_{j}\right)(1+C \delta)>e^{\delta} \beta\left(\xi_{j}\right)
$$

if $\delta$ is sufficiently small, i.e. $N$ sufficiently large, since $C>1$. This contradicts (3.2) and proves Lemma 1 . We deduce

Lemma 2. Suppose that $0<\theta<1$ and $C>0$. Then for $x$ on a set of lower density at least $(1-\theta) /(1-\theta+\theta C)$ we have

$$
\beta(x+h)>\theta \beta(x) \text { for } 0 \leqslant h \leqslant C \theta \beta(x) .
$$

We note that $\beta(x)$ is continuous except on the countable set of jump increases of $f^{(n)}(x)$, where $\beta(x)$ has a jump decrease. At these points we define $\beta(x)=\beta(x+0)$, so that $\beta(x)$ is continuous to the right. We suppose $x_{0}=x_{0}^{\prime}$ to be as in Lemma 1 , and if $x_{j-1}^{\prime}$ has already been defined, we define $x_{j}$ to be the lower bound and so the least value of $x \geqslant x_{j-1}^{\prime}$ such that

$$
\beta(x+h) \leqslant \theta \beta(x) \text { for some } h \leqslant C \theta \beta(x) .
$$


We then choose the least such $h$ and set $x_{j}^{\prime}=x_{j}+h$. Let $E$ be the set of all $x$ in the union of the intervals $\left(x_{j}^{\prime}, x_{j+1}\right)$. Then it is evident that (3.3) holds in $E$. It remains to estimate the lower density of $E$.

Suppose then that $X>x_{0}$, and assume first that $X=x_{p}^{\prime}$ for some $p>0$. Since $x-\beta(x)$ is nondecreasing we note that

$$
\sum_{j=0}^{p}\left\{x_{j}^{\prime}-x_{j}-\beta\left(x_{j}^{\prime}\right)+\beta\left(x_{j}\right)\right\} \leqslant X-x_{0}-\beta(X)+\beta\left(x_{0}\right)<X+O(1) .
$$

Again by our construction

$$
\beta\left(x_{j}\right)-\beta\left(x_{j}^{\prime}\right) \geqslant(1-\theta) \beta\left(x_{j}\right) \geqslant \frac{(1-\theta)}{\theta C}\left(x_{j}^{\prime}-x_{j}\right) .
$$

Thus

$$
\left\{1+\frac{1-\theta}{\theta C}\right\} \sum_{j=0}^{p}\left(x_{j}^{\prime}-x_{j}\right)<X+O(1) .
$$

So if $E(X)=E \cap\left[x_{0}, X\right]$ and $|E(X)|$ denotes the length of $E(X)$ we see that

$$
|E(X)| \geqslant X\left\{1-\frac{C \theta}{C \theta+(1-\theta)}\right\}+O(1)=\frac{(1-\theta)}{C \theta+1-\theta} X+O(1) .
$$

Next if $x_{p} \leqslant X \leqslant x_{p}^{\prime}, X$ is smaller while $|E(X)|$ is the same, so that (3.5) is still valid. Again if $x_{p}^{\prime} \leqslant X<x_{p+1}, X$ is larger, so that (3.4) and (3.5) are still valid. Thus (3.5) holds in all cases and Lemma 2 is proved.

LEMMA 3. Suppose that for some numbers $x=x_{0}, \theta$ and $C$ we have (3.3). Then

$$
f\left\{x_{0}+C \theta \beta\left(x_{0}\right)\right\} \leqslant\left\{\beta\left(x_{0}\right)\right\}^{n} e^{C} f^{(n)}\left(x_{0}\right) .
$$

We write

$$
\beta=\theta \beta\left(x_{0}\right), \quad \alpha=(\beta / \theta)^{n} f^{(n)}\left(x_{0}\right), \quad \varphi(x)=\alpha \exp \left\{\left(x-x_{0}\right) / \beta\right\},
$$

and suppose that (3.6) is false. From this we shall obtain a contradiction to (3.3).

We define

$$
x_{2}=\inf \left\{x, x_{0} \leqslant x \text { and for some } \nu, 0 \leqslant \nu \leqslant n, f^{(\nu)}(x)>\varphi^{(\nu)}(x)\right\} .
$$

Since (3.6) is false we have

$$
\varphi\left(x_{0}+C \beta\right)=\alpha e^{C}=(\beta / \theta)^{n} e^{C} f^{(n)}\left(x_{0}\right)<f\left(x_{0}+C \beta\right) .
$$

Again for $\nu=0, \ldots, n$ we have

$$
\varphi^{(\nu)}\left(x_{0}\right)=\alpha / \beta^{\nu}=\theta^{-n} \beta^{n-\nu} f^{(n)}\left(x_{0}\right) \geqslant \beta\left(x_{0}\right)^{n-\nu} f^{(n)}\left(x_{0}\right) \geqslant f^{(\nu)}\left(x_{0}\right) .
$$

Thus $x_{2}$ exists and $x_{0} \leqslant x_{2}<x_{0}+C \beta$.

Suppose now that for some $\nu<n$ we have

$$
\varphi^{(\nu)}\left(x_{2}\right) \leqslant f^{(\nu)}\left(x_{2}\right) .
$$

Then we have by the definition of $x_{2}$

$$
\varphi^{(\nu)}(x) \geqslant f^{(\nu)}(x), \quad 0 \leqslant x<x_{2} .
$$


Hence we deduce that

$$
\frac{d}{d x} \frac{\varphi^{(\nu)}(x)}{f^{(\nu)}(x)} \leqslant 0
$$

at $x=x_{2}$, where differentiation denotes the left derivative. Thus

$$
1 \leqslant \frac{f^{(\nu)}\left(x_{2}\right)}{\varphi^{(\nu)}\left(x_{2}\right)} \leqslant \frac{f^{(\nu+1)}\left(x_{2}\right)}{\varphi^{(\nu+1)}\left(x_{2}\right)},
$$

so that (3.7) holds with $\nu+1$ instead of $\nu$. Thus finally (3.7) must hold with $\nu=n$ for the left derivative and so also the right derivative, while by the definition of $x_{2}$ we have (3.8) for $\nu<n$ and $x<x_{2}$ and by continuity also for $x=x_{2}$. Thus

$$
\frac{f^{(\nu)}\left(x_{2}\right)}{f^{(n)}\left(x_{2}\right)} \leqslant \frac{\varphi^{(\nu)}\left(x_{2}\right)}{\varphi^{(n)}\left(x_{2}\right)}=\beta^{n-\nu}, \quad \nu=0, \ldots, n-1,
$$

so that $\beta\left(x_{2}\right) \leqslant \beta=\theta \beta\left(x_{0}\right)$. This contradicts (3.3) and so Lemma 3 is proved.

We can now complete the proof of Theorem 3. We set $h=C \theta \beta\left(x_{0}\right)$ and deduce from (3.6) that if (3.3) holds with $x=x_{0}$ then

$$
f_{n}\left(x_{0}\right) \leqslant \frac{f\left(x_{0}+h\right)}{h^{n}} \leqslant \frac{e^{C}}{(C \theta)^{n}} f^{(n)}\left(x_{0}\right) .
$$

By Lemma 2 we deduce that this inequality holds in a set of lower density at least $\delta=(1-\theta) /(1-\theta+\theta C)$. Setting $C=n, \theta=K^{-1}$ we deduce Theorem 3.

4. Proof of Theorem 1. In this section we suppose that $u(z)$ is subharmonic and not constant in the plane and that $u(0)=0$. We write

$$
\begin{gathered}
B(r)=\sup _{|=|=r} u(z), \\
b(r)=\int_{0}^{r}(r-t) B(t) d t, \quad b_{2}(r)=\inf _{h>0} \frac{b(r+h)}{h^{2}},
\end{gathered}
$$

so that $b^{\prime \prime}(r)=B(r)$. We also write $n(z, h)$ for the Riesz mass of $u$ in the disk $|\zeta-z| \leqslant h$ and set

$$
\begin{gathered}
N(z, h)=\int_{0}^{h} \frac{n(z, t) d t}{t}, \\
u(z, h)=u(z)+N(z, h)=\frac{1}{2 \pi} \int_{0}^{2 \pi} u\left(z+h e^{i \theta}\right) d \theta .
\end{gathered}
$$

Suppose that $f(z)$ is a transcendental entire function and that $a$ is a complex constant. Then we have

$$
f(z)-a=c_{\lambda} z^{\lambda}+\cdots
$$

and will apply our results to

$$
u_{a}(z)=\log \left|\frac{(f(z)-a)}{c_{\lambda} z^{\lambda}}\right|=\log \left|f_{a}(z)\right| .
$$

We denote by $A_{1}, A_{2}, A_{3}, \ldots$ positive absolute constants. We need 
LEMMA 4. If $0<|z|=r<R$ and $h=A_{1}(R-r), 0<A<1$, we have

$$
u\left(z, \frac{1}{2} h\right)>-\frac{A_{2}}{(R-r)^{2}} b(R)
$$

and

$$
n(z, h)<\frac{A_{3}}{(R-r)^{2}} b(R) .
$$

Further if $0<d<\frac{1}{2} h$ we have

$$
N\left(\zeta, \frac{1}{2} h\right)<\frac{A_{4}}{(R-r)^{2}} \log \left(\frac{16 h}{d}\right) b(R)
$$

for $|\zeta-z|<\frac{1}{2} h$ except possibly when $\zeta$ lies in a set of disks, the sum of whose radii is at most $d$.

The conclusions (4.7)-(4.9) are (14.1)-(14.3) of [2, p. 494]. The quantity $b(r)$ of the present paper is the $B_{2}(r)$ of [2].

We now prove

THEOREM 4. With the above notation there exists an absolute constant $A_{5}$, such that if $K>0$, we have

$$
u\left(r e^{i \theta}\right)>-K b_{2}(r)
$$

for $0 \leqslant \theta \leqslant 2 \pi$, outside a set $e(r, K)$ of $\theta$ whose measure is at most $4 \pi \exp \left(-A_{5} K\right)$.

We start by finding $R$, such that $r<R \leqslant 2 r$ and

$$
b(R) /(R-r)^{2} \leqslant 4 b_{2}(r) .
$$

If $R>2 r$, we deduce from the fact that $B(r)$ increases with $r$ that so does

$$
R^{-2} b(R)=\int_{0}^{1}(1-t) B(R t) d t .
$$

Hence for $R>2 r$

$$
\frac{b(R)}{(R-r)^{2}} \geqslant \frac{b(R)}{R^{2}} \geqslant \frac{b(2 r)}{(2 r)^{2}}=\frac{1}{4} \frac{b(2 r)}{(2 r-r)^{2}} .
$$

Thus

$$
\inf _{R \geqslant 2 r} \frac{b(R)}{(R-r)^{2}} \geqslant \frac{1}{4} \frac{b(2 r)}{(2 r-r)^{2}}
$$

and so

$$
b_{2}(r) \geqslant \frac{1}{4} \min _{r<R \leqslant 2 r} \frac{b(R)}{(R-r)^{2}} .
$$

Thus $R$ exists satisfying (4.11). Having chosen $R$ to satisfy (4.11) we define $h$ as in Lemma 4 and apply that lemma. We define $p$ to be the smallest integer such that $p \geqslant 2$ and

$$
2 \sin (\pi / 2 p)=|\exp (\pi i / p)-1|<\frac{1}{2} h / r .
$$


Then if $z_{\nu}=r \exp (2 \pi i \nu / p)$, the disks $C_{\nu}:\left|z-z_{\nu}\right|<\frac{1}{2} h, \nu=1, \ldots, p$, cover $|z|=r$. Also

$$
2 \pi / p \geqslant \pi /(p-1) \geqslant 2 \sin (\pi / 2(p-1)) \geqslant \frac{1}{2} h / r,
$$

so that $p \leqslant 4 \pi r / h$.

Again for $d<\frac{1}{2} h$ we have (4.9) in $C_{\nu}$ outside a set $E_{\nu}$ of disks the sum of whose radii is at most $d$. Since $d<\frac{1}{2} h<\frac{1}{2} r$ each exceptional disk $\left|z-z_{j}\right|<d_{j} \leqslant d$ meets $z=r e^{i \theta}$ in an arc of diameter at most $2 d_{j}$ and so length at most $\pi d_{j}$. Thus the total length of those arcs on $C_{\nu} \cap(|z|=r)$, which lie in the exceptional disks is at most $\pi d$. Thus (4.9) holds on $|\zeta|=r$, outside a set of arcs of total length at most $\pi p d$, i.e. (4.9) holds for $\zeta=r e^{i \theta}, 0 \leqslant \theta \leqslant 2 \pi$, except for a set $e(r)$ of $\theta$ having measure

$$
|e(r)| \leqslant \pi p d / r \leqslant(\pi d / r)(4 \pi r / h)=4 \pi^{2} d / h .
$$

Further, for $\theta$ outside $e(r)$ we have from (4.4), (4.7) and (4.9)

$$
\begin{aligned}
u\left(r e^{i \theta}\right) & =u\left(r e^{i \theta}, \frac{1}{2} h\right)-N\left(r e^{i \theta}, \frac{1}{2} h\right) \\
& >\frac{-b(R)}{(R-r)^{2}}\left(A_{2}+A_{4} \log \frac{16 h}{d}\right)>-b_{2}(r)\left(4 A_{2}+4 A_{4} \log \frac{16 h}{d}\right)
\end{aligned}
$$

by (4.11). Suppose now that $K>4 A_{2}+4 A_{4} \log 32$. Then we define $d<\frac{1}{2} h$ by $K=4 A_{2}+4 A_{4} \log (16 h / d)$ and deduce from (4.12) and (4.13) that (4.10) holds outside a set $\theta$ of measure

$$
|e(r, K)| \leqslant 4 \pi^{2} d / h=64 \pi^{2} \exp \left(A_{2} / A_{4}-K / 4 A_{4}\right) \leqslant \exp \left(-K / 8 A_{4}\right)
$$

if $K \geqslant A_{6}$. This proves Theorem 4 for $K \geqslant A_{6}$. Also, if $K<A_{6},(4.10)$ is trivial if $\exp \left(A_{5} A_{6}\right)<2$. Thus Theorem 4 holds in all cases with $A_{5}=\inf \left(1 / 8 A_{4},(\log 2) / A_{6}\right)$.

We deduce the following consequence from Theorem 4 , which may be considered as an analogue of the Edrei-Fuchs small arcs lemma [1, p. 322].

THEOREM 5. If $E$ is a set of measure $\delta<2 \pi$ on the interval $[0,2 \pi]$ then we have

$$
\int_{E} u\left(r e^{i \theta}\right) d \theta>-A_{7} b_{2}(r) \delta \log \left(\frac{4 \pi}{\delta}\right) .
$$

We denote by $e(K)$ the set of $\theta$ such that $u\left(r e^{i \theta}\right)<-K b_{2}(r)$ and by $m(K)$ the measure of $e(K)$. Then Theorem 4 gives

$$
\begin{aligned}
\int_{e(K)} u\left(r e^{i \theta}\right) d \theta & =b_{2}(r) \int_{K}^{\infty} t d m(t)=-b_{2}(r)\left\{K m(K)+\int_{K}^{\infty} m(t) d t\right\} \\
& >-4 \pi b_{2}(r)\left\{K \exp \left(-A_{5} K\right)+\int_{K}^{\infty} \exp \left(-A_{5} t\right) d t\right\} \\
& >-A_{9} b_{2}(r) \exp \left(-A_{8} K\right) .
\end{aligned}
$$

Given $E$ as in Theorem 5 we choose $K>0$, and define $E_{1}, E_{2}$ to be the subsets of $E$, where $u<-K b_{2}(r), u \geqslant-K b_{2}(r)$, respectively. Then

$$
\begin{aligned}
\int_{E} u\left(r e^{i \theta}\right) d \theta & =\int_{E_{1}}+\int_{E_{2}} \geqslant \int_{e(K)} u\left(r e^{i \theta}\right) d \theta+\int_{E_{2}} u\left(r e^{i \theta}\right) d \theta \\
& \geqslant-b_{2}(r)\left\{A_{9} \exp \left(-A_{8} K\right)+K \delta\right\}
\end{aligned}
$$


We choose $K$ so that $A_{9} \exp \left(-A_{8} K\right)=\delta$, i.e. $K=\left(A_{8}\right)^{-1} \log \left(A_{9} / \delta\right)$, and deduce that

$$
\int_{E} u\left(r e^{i \theta}\right) d \theta>-b_{2}(r) \delta\left\{1+\frac{1}{A_{8}} \log \frac{A_{9}}{\delta}\right\}
$$

which gives Theorem 5 .

We can now complete the proof of Theorem 1. Suppose that we have on the set $E$ of values of $r$

$$
T(r, f)>\left(1-\varepsilon(r)^{2}\right) \log M(r, f)
$$

where

$$
\varepsilon(r) \rightarrow 0, \text { but } \varepsilon(r)^{2} \log M(r) / \log r \rightarrow \infty
$$

as $r \rightarrow \infty$. We define $u(z)=\log |f(z)|$.

Let $F$ be the set of all $r$, such that

$$
b_{2}(r)>\frac{1}{\varepsilon(r)} B(r)=\frac{1}{\varepsilon(r)} b^{\prime \prime}(r), \quad \text { where } B(r)=\log M(r, f) .
$$

Then given $K>1$, we have for all large $r$ in $F$

$$
b_{2}(r) \geqslant \frac{e^{2} K^{2}}{4} b^{\prime \prime}(r),
$$

so that $F$ has upper density at most $2 /(K+1)$ by Theorem 3 . Since $K$ is arbitrary, $F$ has density zero.

Suppose now that $a$ is any complex number and replace $u(z)$ by the function $u_{a}(z)$ defined by (4.6). Then

$$
u_{a}(z)=\log |f(z)-a|+O(\log |z|)
$$

so that for $|z|=r$

$$
u_{a}^{+}(z)=\max \left(u_{a}(z), 0\right)=\left\{\log ^{+}|f(z)|+O(\log r)\right\} .
$$

Thus, since $f(z)$ is transcendental we have $B\left(r, u_{a}(z)\right)=B(r)+O(\log r)$ as $r \rightarrow \infty$, and similarly $T\left(r, f_{a}(z)\right)=T(r, f)+O(\log r)$. Hence, also we have as $r \rightarrow \infty$

$$
b\left(r, u_{a}\right) \sim b(r), \quad b_{2}\left(r, u_{a}\right) \sim b_{2}(r) .
$$

We deduce from (4.16) that for any complex $a$ we have for $r \in E \backslash F$ and $r>r_{0}(a)$

$$
b_{2}\left(r, u_{a}\right)<\frac{2}{\varepsilon(r)} B\left(r, u_{a}\right)
$$

and from (4.14) and (4.15) that

$$
T\left(r, f_{a}\right)>\left\{1-2 \varepsilon(r)^{2}\right\} B\left(r, u_{a}\right) .
$$

Suppose now that for such a value of $r, e(r, a)$ is the set of all $\theta$ for which $u_{a}<0$ and let $e^{\prime}(r, a)$ be the complementary set of $\theta$. Then

$$
2 \pi T\left(r, f_{a}\right)=\int_{0}^{2 \pi} u_{a}^{+}\left(r e^{i \theta}\right) d \theta=\int_{,}+\int_{e^{\prime}} \leqslant(2 \pi-|e(r, a)|) B\left(r, u_{a}\right),
$$


where $|e|$ denotes the measure of $e$. Thus

$$
T\left(r, f_{a}\right) \leqslant\left(1-\frac{|e(r, a)|}{2 \pi}\right) B\left(r, u_{a}\right),
$$

so that by (4.18), $|e(r, a)| \leqslant 4 \pi \varepsilon(r)^{2}$. Thus Theorem 5 and (4.17) yield for large $r$ in $E \backslash F$

$$
\begin{aligned}
m(r, a)+O(\log r) & =\frac{-1}{2 \pi} \int_{e(r, a)} u_{a}\left(r e^{i \theta}\right) d \theta \\
& <A_{7} b_{2}\left(r, u_{a}\right)|e(r, a)| \log \frac{4 \pi}{|e(r, a)|} \\
& =O\left\{B\left(r, u_{a}\right) \varepsilon(r) \log \frac{1}{\varepsilon(r)}\right\}=o\{T(r, f)\},
\end{aligned}
$$

and this proves Theorem 1 , for $E \backslash F$ has positive upper density and so is unbounded.

5. Another growth lemma. In order to prove Theorem 2 we need

LEMMA 5. Suppose that $B(r)$ is a positive increasing function of positive order, that $b(r)$ and $b_{2}(r)$ are defined by (4.2) and that $\varphi(r)$ is a positive function of $r$, such that

$$
\varphi(r)=O\left\{b_{2}(r)\right\} \quad \text { as } r \rightarrow \infty
$$

and for some function $\varepsilon(r)$, which decreases to zero as $r \rightarrow \infty$, we have

$$
\varphi(r)=O\left\{\varepsilon(r) b_{2}(r)\right\} \quad \text { as } r \rightarrow \infty
$$

on a set $E_{1}$ of density one. Then there exists a set $E_{2}$ of upper density one, depending only on $E_{1}$ and the function $\varepsilon(r)$, such that

$$
\int_{1}^{r} \varphi(t) \log \left(\frac{r}{t}\right) \frac{d t}{t}=o\{B(r)\} \quad \text { as } r \rightarrow \infty
$$

in $E_{2}$.

We note that $b(r)$ and $b_{2}(r)$ also increase with $r$, and have positive order. In fact, the increasing property is obvious from (4.2) and

$$
h^{-2} b(r+h) \geqslant h^{-2} \int_{r}^{r+h}(r+h-t) B(t) d t \geqslant \frac{1}{2} B(r)
$$

so that $b_{2}(r) \geqslant \frac{1}{2} B(r)$ and $b(2 r) \geqslant r^{2} B(r) / 2$ for all $r$. Thus if $B(r)$ has positive order $\lambda, b(r)$ has order at least $\lambda+2$ and $b_{2}(r)$ has order at least $\lambda$. We now choose $\mu$ such that $0<\mu<\lambda$ and a sequence $R_{n}$, which tends to $\infty$ with $n$ and is such that

$$
b_{2}(r) \leqslant\left(r / R_{n}\right)^{\mu} b_{2}\left(R_{n}\right) \text { for } 1 \leqslant r<R_{n} .
$$

Since $b_{2}(r) / r^{\mu}$ is continuous and unbounded we may for instance choose $R_{1}=1$ and if $R_{n-1}$ has been defined let $R_{n}$ be the smallest number such that $R_{n} \geqslant 2 R_{n-1}$ and

$$
b_{2}\left(R_{n}\right) / R_{n}^{\mu} \geqslant \sup _{1 \leqslant R \leqslant 2 R_{n-1}} b(r) / r^{\mu}
$$


We proceed to show that if $K_{n}$ tends to $\infty$ sufficiently slowly with $n$ and $E_{2}$ consists of all those points $r$ in the intervals $\left[R_{n}, K_{n} R_{n}\right]$ for which

$$
b_{2}(r)<K_{n}^{\mu / 2} B(r),
$$

then the set $E_{2}$ has the required property.

We note first that $E_{2}$ has upper density one. In fact, it follows from Theorem 3 that given $K>1$ we have

$$
b_{2}(r)<(e K / 2)^{2} B(r)
$$

for a set of $r$ in $\left[0, K_{n} R_{n}\right]$ having measure at least $(K-1) K_{n} R_{n} /(K+1)+O(1)$ when $R_{n}$ is large and so in a set in $\left[R_{n}, K_{n} R_{n}\right]$ having measure at least

$$
\left\{\frac{K-1}{K+1}-\frac{1}{K_{n}}\right\} K_{n} R_{n}+O(1) .
$$

Thus, since (5.6) implies (5.5) for large $n$, we see that $E_{2}$ has upper density at least $(K-1) /(K+1)$, and since $K$ can be as large as we please $E_{2}$ has upper density one.

We next choose the quantities $K_{n}$. Let $E_{1}^{\prime}$ be the complement of $E_{1}$, let $E_{1}^{\prime}[r]$ be the intersection of $E_{1}^{\prime}$ with the interval $[0, r]$, and let $\left|E_{1}^{\prime}[r]\right|$ be the measure of $E_{1}^{\prime}[r]$. Then we assume that $K_{n}$ tends to infinity so slowly that

$$
K_{n}^{2+\mu}<r /\left|E_{1}^{\prime}(r)\right|, \quad r \geqslant R_{n} .
$$

This is possible since $E_{1}^{\prime}$ has density zero and $R_{n} \rightarrow \infty$ with $n$. We also assume that

$$
K_{n}^{\mu} \varepsilon\left(R_{n} / K_{n}\right)<1,
$$

which is possible since $\varepsilon(r) \rightarrow 0$ as $r \rightarrow \infty$. The set $E_{2}$ defined as above is independent of $\varphi(r)$ and has upper density one. It remains to show that (5.3) holds in $E_{2}$.

Assume that $r \in E_{2}, R_{n} \leqslant r \leqslant K_{n} R_{n}$, and write

$$
I(r)=\int_{1}^{r} \varphi(t) \log \frac{r}{t} \frac{d t}{t}=I_{0}(r)+I_{1}(r)+I_{1}^{\prime}(r),
$$

where $I_{0}(r), I_{1}(r)$ and $I_{1}^{\prime}(r)$ are the integrals over the ranges $\left[1, R_{n} / K_{n}\right], e_{1}=$ $\left[R_{n} / K_{n}, r\right] \cap E_{1}$ and $e_{1}^{\prime}=\left[R_{n} / K_{n}, r\right] \cap E_{1}^{\prime}$, respectively. Then by (5.1), (5.4) and (5.5) we have

$$
\begin{aligned}
& I_{0}(r)=\int_{1}^{R_{n} / K_{n}} \varphi(t) \log \frac{r}{t} \frac{d t}{t} \leqslant 2 \int_{1}^{R_{n} / K_{n}} \varphi(t) \log \frac{R_{n}}{t} \frac{d t}{t} \\
& =O\left\{\int_{1}^{R_{n} / K_{n}} b_{2}(t) \log \frac{R_{n}}{t} \frac{d t}{t}\right\}=O\left\{b_{2}\left(R_{n}\right) \int_{1}^{R_{n} / K_{n}}\left(\frac{t}{R_{n}}\right)^{\mu} \log \frac{R_{n}}{t} \frac{d t}{t}\right\} \\
& =O\left\{b_{2}\left(R_{n}\right) K_{n}^{-\mu} \log \frac{1}{K_{n}}\right\}=O\left(b_{2}(r) K_{n}^{-\mu} \log \frac{1}{K_{n}}\right) \\
& =O\left\{B(r) K_{n}^{-\mu / 2} \log \frac{1}{K_{n}}\right\}=o\{B(r)\} \text {. }
\end{aligned}
$$


Again by (5.2), (5.5) and (5.8)

$$
\begin{aligned}
I_{1}(r) & =O\left\{\varepsilon\left(\frac{R_{n}}{K_{n}}\right) \int_{e_{1}} b_{2}(t) \log \frac{r}{t} \frac{d t}{t}\right\} \\
& =O\left\{b_{2}(r) \varepsilon\left(\frac{R_{n}}{K_{n}}\right) \int_{r / K_{n}^{2}}^{r} \log \frac{r}{t} \frac{d t}{t}\right\} \\
& =O\left\{b_{2}(r) \varepsilon\left(\frac{R_{n}}{K_{n}}\right)\left(\log K_{n}\right)^{2}\right\} \\
& =O\left\{B(r) \varepsilon\left(\frac{R_{n}}{K_{n}}\right) K_{n}^{\mu / 2}\left(\log K_{n}\right)^{2}\right\}=o\{B(r)\} .
\end{aligned}
$$

Finally, by (5.1), (5.5) and (5.7)

$$
\begin{aligned}
I_{1}^{\prime}(r) & =O\left\{\int_{e_{1}^{\prime}} b_{2}(t) \log \frac{r}{t} \frac{d t}{t}\right\}=O\left\{b_{2}(r)\left(\frac{K_{n}^{2}}{r}\right) \log K_{n} \int_{e_{1}^{\prime}} d t\right\} \\
& =O\left\{B(r) K_{n}^{2+\mu / 2}\left(\log K_{n}\right) r^{-1}\left|E_{1}^{\prime}(r)\right|\right\}=o\{B(r)\} .
\end{aligned}
$$

Now (5.3) follows from (5.9) and Lemma 5 is proved.

6. Proof of Theorem 2. In order to prove Theorem 2 we need a formalism used elsewhere. We suppose that $f(z)$ is a transcendental entire function such that $f(0)=1$ and denote by $n\left(r, \theta_{1}, \theta_{2}\right)$ the number of zeros of $f(z)$ in $0<|z|<r$, $\theta_{1}<\arg z<\theta_{2}$ each counted with due multiplicity. We also write

$$
N\left(r, \theta_{1}, \theta_{2}\right)=\int_{0}^{r} n\left(t, \theta_{1}, \theta_{2}\right) \frac{d t}{t} .
$$

Next, if $f(z) \neq 0$ on the segment $z=t e^{i \theta}, 0 \leqslant t \leqslant r$, we define $v(t, \theta)$ to be the continuous value of $\arg f(z)$ on this segment such that $v(0, \theta)=0$, and we write,

$$
V(r, \theta)=\frac{1}{2 \pi} \int_{0}^{r} v(t, \theta) \frac{d t}{t} .
$$

With this notation we have [3, Theorem 1]

LEMMA 6. If $f(z) \neq 0$ on the segments $z=t e^{i \theta}, 0 \leqslant t \leqslant r, \theta=\theta_{1}$ or $\theta_{2}$, then

$$
N\left(r, \theta_{1}, \theta_{2}\right)=\frac{1}{2 \pi} \int_{\theta_{1}}^{\theta_{2}} \log \left|f\left(r e^{i \theta}\right)\right| d \theta+V\left(r, \theta_{1}\right)-V\left(r, \theta_{2}\right) .
$$

We need to transform the quanity $V(r, \theta)$ a little and note that

$$
v(r, \theta)=\int_{0}^{r} \frac{\partial v(t, \theta)}{\partial t} d t=\int_{0}^{r}-\frac{1}{t} \frac{\partial}{\partial \theta} \log \left|f\left(t e^{i \theta}\right)\right| d t .
$$

Thus, for $\alpha<\beta \leqslant \alpha+2 \pi$ we have

$$
\begin{aligned}
\int_{\alpha}^{\beta} V(r, \theta) d \theta & =-\frac{1}{2 \pi} \int_{0}^{r} \frac{d s}{s} \int_{0}^{s} \frac{d t}{t} \int_{\alpha}^{\beta} \log \left|f\left(t e^{i \theta}\right)\right| d \theta \\
& =\frac{1}{2 \pi} \int_{0}^{r}\left\{\log \left|f\left(t e^{i \alpha}\right)\right|-\log \left|f\left(t e^{i \beta}\right)\right|\right\} \log \frac{r}{t} \frac{d t}{t} .
\end{aligned}
$$


We write

$$
M(t)=\sup _{0 \leqslant \theta \leqslant 2 \pi}\left|f\left(t e^{i \theta}\right)\right|, \quad B(t)=\log M(t)
$$

and

$$
\varphi(t, \alpha)=\frac{1}{2 \pi} \log \frac{M(t)}{\left|f\left(t e^{i \alpha}\right)\right|}
$$

Thus

$$
\int_{\alpha}^{\beta} V(r, \theta) d \theta=\int_{0}^{r}\{\varphi(t, \beta)-\varphi(t, \alpha)\} \log \frac{r}{t} \frac{d t}{t} .
$$

Our aim is to show that the positive function $\varphi(t, \alpha)$ is on the average not too large. We also define $\varphi_{a}(t, \alpha)$ to be the function $\varphi(t, \alpha)$ defined as above w.r.t. the functions $f_{a}(z)$ introduced in (4.6).

LEMMA 7. If

$$
\varphi_{a}(t)=\frac{1}{2 \pi} \int_{0}^{2 \pi} \varphi_{a}(t, \alpha) d \alpha,
$$

then under the hypotheses of Theorem 2 there exists a set $E_{2}$ of upper density one such that we have

$$
\int_{1}^{r} \varphi_{a}(t) \log \frac{r}{t} \frac{d t}{t}=o\{B(r)\}
$$

as $r \rightarrow \infty$ in $E_{2}$ for each complex $a$.

We define the set $E_{2}$ as in Lemma 5, where $B(r)$ is given by (6.1) (i.e. for the function $f_{0}(z)=f(z)$ ). Then $B(r)$ has positive order by hypothesis, so that Lemma 5 is applicable. We deduce from Theorem 1 that under the hypotheses of Theorem 1 , we have for each complex $a$ as $r \rightarrow \infty$ in $E_{1}$

$$
\begin{aligned}
\frac{1}{2 \pi} \int_{0}^{2 \pi} \log ^{+}\left|f_{a}\left(r e^{i \theta}\right)\right| d \theta & =\frac{1}{2 \pi} \int_{0}^{2 \pi} \log ^{+}\left|f\left(r e^{i \theta}\right)\right|+O(\log r) \\
& >B(r)-\varepsilon(r) B(r)+O(\log r),
\end{aligned}
$$

where $\varepsilon(r) \rightarrow 0$ with $r$ and $\varepsilon(r)$ is independent of $a$. Thus $\left|f_{a}\left(r e^{i \theta}\right)\right|>1$ outside a set of $\theta$ of measure at most $O\{\varepsilon(r)\}$, provided that $\varepsilon(r) \rightarrow 0$ so slowly that $\log r=$ $o\{\varepsilon(r) B(r)\}$ as $r \rightarrow \infty$. Now Theorem 5 shows that as $r \rightarrow \infty$ in $E_{1}$ we have

$$
\begin{aligned}
\int_{0}^{2 \pi} \log ^{+}\left|\frac{1}{f_{a}\left(r e^{i \theta}\right)}\right| d \theta & =O\left\{\varepsilon(r) \log \frac{1}{\varepsilon(r)} b_{2}(r)\right\} \\
& =O\left\{\varepsilon_{1}(r) b_{2}(r)\right\},
\end{aligned}
$$

where $\varepsilon_{1}(r)$ is independent of $a$. We note that if $a \neq 1, f_{a}(z)=(f(z)-a) /(1-a)$ while $f_{1}(z)=(f(z)-1) / z^{\lambda}$. Thus for any fixed $a$ and large $r$ we have

$$
M_{a}(r)=\sup _{|z|=r}\left|f_{a}(z)\right|<C_{a} M(r), \quad|z|=r,
$$


where the constant $C_{a}$ depends only on $a$. Also (6.3) and (6.4) yield

$$
\begin{aligned}
\frac{1}{2 \pi} \int_{0}^{2 \pi} \log \frac{M_{a}(r)}{\left|f_{a}\left(r e^{i \theta}\right)\right|} d \theta & =O\left\{\varepsilon(r) B(r)+\varepsilon_{1}(r) b_{2}(r)+\log r\right\} \\
& =O\left\{\varepsilon_{1}(r) b_{2}(r)\right\}
\end{aligned}
$$

as $r \rightarrow \infty$ in $E_{1}$. Thus, if $\varphi_{a}(t)$ is defined with $f_{a}(z)$ instead of $f(z)$, we see that for each complex $a$

$$
\varphi_{a}(t)=\frac{1}{2 \pi} \int_{0}^{2 \pi} \varphi_{a}(t, \alpha) d t=O\left\{\varepsilon_{1}(t) b_{2}(t)\right\}
$$

as $t \rightarrow \infty$ in $E_{1}$, while we have in any case

$$
\varphi_{a}(t)=B(t)-\frac{1}{2 \pi} \int_{0}^{2 \pi} \log \left|f_{a}\left(t e^{i \theta}\right)\right| d \theta+O(1) \leqslant B(t)+O(1)=O\left\{b_{2}(t)\right\} .
$$

Thus $\varphi_{a}(t)$ satisfies the hypotheses of Lemma 5 and we deduce that there exists a set $E_{2}$ of upper density one such that for each complex $a$ we have

$$
\int_{1}^{r} \varphi_{a}(t) \log \frac{r}{t} \frac{d t}{t}=o\{B(r)\} \quad \text { as } r \rightarrow \infty \text { on } E_{2} .
$$

This proves Lemma 7.

We now suppose that we are given $\varepsilon>0$ and $\theta_{1}, \theta_{2}$, such that $\theta_{1} \leqslant \theta_{2}<\theta_{1}+2 \pi$. We also take a fixed complex $a$ and assume that $r \rightarrow \infty$ on $E_{2}$. Then there exist $\alpha, \beta$ such that $\theta_{2}<\alpha<\theta_{2}+\varepsilon / 3, \theta_{2}+2 \varepsilon / 3<\beta<\theta_{2}+\varepsilon$ and

$$
\begin{aligned}
0 & \leqslant \int_{1}^{r} \varphi_{a}(t, \alpha) \log \left(\frac{r}{t}\right) \frac{d t}{t} \leqslant \frac{3}{\varepsilon} \int_{1}^{r} \log \frac{r}{t} \frac{d t}{t} \int_{\theta_{2}}^{\theta_{2}+\varepsilon / 3} \varphi_{a}(t, \theta) d \theta \\
& \leqslant \frac{6 \pi}{\varepsilon} \int_{1}^{r} \log \frac{r}{t} \varphi_{a}(t) \frac{d t}{t}, \\
0 & \leqslant \int_{1}^{r} \varphi_{a}(t, \beta) \log \frac{r}{t} \frac{d t}{t} \leqslant \frac{6 \pi}{\varepsilon} \int_{1}^{r} \log \varphi_{a}(t) \frac{d t}{t} .
\end{aligned}
$$

Using (6.2) and (6.5) we deduce that $\left|\int_{\alpha}^{\beta} V(r, \theta) d \theta\right|=o\{B(r)\}$. Hence there exists $\varphi_{2}=\varphi_{2}(r)$ such that $\alpha<\varphi_{2}<\beta$ and so $\theta_{2}<\varphi_{2}<\theta_{2}+\varepsilon$ and $V\left(r, \varphi_{2}\right)=o\{B(r)\}$. Similarly, there exists $\varphi_{1}$, such that $\theta_{1}-\varepsilon<\varphi_{1}<\theta_{1}$, and $V\left(r, \varphi_{1}\right)=o\{B(r)\}$. Thus Lemma 6 shows that

$$
\begin{aligned}
N\left(r, \theta_{1}, \theta_{2}\right) & \leqslant N\left(r, \varphi_{1}, \varphi_{2}\right) \leqslant \frac{\varphi_{2}-\varphi_{1}}{2 \pi}\{B(r)+O(1)\}+o\{B(r)\} \\
& \leqslant \frac{\theta_{2}-\theta_{1}+2 \varepsilon+o(1)}{2 \pi} B(r) .
\end{aligned}
$$

This gives

$$
\varlimsup \frac{N\left(r, \theta_{1}, \theta_{2}\right)}{B(r)} \leqslant \frac{\theta_{2}-\theta_{1}}{2 \pi .}
$$

Also, we may assume that $E_{2}$ is disjoint from the set $F$ of Theorem 1, since this does not affect the density. Then as $r \rightarrow \infty$ in $E_{2}$

$$
N\left(r, \theta_{2}, \theta_{1}+2 \pi\right)+N\left(r, \theta_{1}, \theta_{2}\right)=N(r)=(1+o(1)) B(r)
$$


by Theorem 1 . We apply (6.6) with $\theta_{2}, \theta_{1}+2 \pi$ instead of $\theta_{1}, \theta_{2}$ and obtain

$$
\varlimsup \frac{N\left(r, \theta_{2}, \theta_{1}+2 \pi\right)}{B(r)} \leqslant \frac{2 \pi+\theta_{1}-\theta_{2}}{2 \pi} .
$$

Now (6.7) gives

$$
\underline{\lim } \frac{N\left(r, \theta_{1}, \theta_{2}\right)}{B(r)} \geqslant \frac{\theta_{2}-\theta_{1}}{2 \pi} .
$$

Combining this with (6.6) we obtain

$$
\lim \frac{N\left(r, \theta_{1}, \theta_{2}\right)}{B(r)}=\frac{\theta_{2}-\theta_{1}}{2 \pi}
$$

as $r \rightarrow \infty$ in $E_{2}$, and this proves Theorem 2 .

In conclusion we note that, by Theorem 2, (1.2) implies angular equidistribution of all $a$-values unless $f(z)$ has order zero. However, for functions of order zero it follows from Theorem 3 of [3] that $f(z)$ satisfies the conclusion of Theorem 2 if (1.4) holds and a fortiori if (1.1) holds. Thus (1.1) always implies equidistribution of the $a$-values.

\section{REFERENCES}

1. A Edrei and W. H. J. Fuchs, Bounds for the number of deficient values of certain classes of meromorphic functions, Proc. London Math. Soc. (3) 12 (1962), 315-344.

2. W. K. Hayman, The minimum modulus of large integral functions, Proc. London Math. Soc. (3) 2 (1952), 469-512.

3. , Angular value distribution of power series with gaps, Proc. London Math. Soc. (3) 24 (1972), $590-624$.

4. 115-145.

5. W. K. Hayman and F. M. Stewart, Real inequalities with applications to function theory, Proc. Cambridge Philos. Soc. 50 (1954), 250-260.

6. T. Murai, The deficiency of entire functions with Fejér gaps, Ann. Inst. Fourier (Grenoble) 33 (1983), $39-58$.

Department of Mathematics, Imperial COllege, London SW7 2BZ, England

Department of Mathematics, Virginia Polytechnic Institute and State University, BlacksBURG, VIRGINIA 24061 